\title{
Bio-Guided Targeting for Preservative and Anti-Ageing Cosmetic Ingredient Development
}

\author{
Emilie Destandau $^{1, *}$, Isabelle Krolikiewicz-Renimel ${ }^{2}$, Saida El Abdellaoui ${ }^{1}$, Perrine Cancellieri ${ }^{3}$, \\ Laetitia Fougère ${ }^{1}$, Alix Toribio ${ }^{1}$, Ludovic Landemarre ${ }^{3}$, Patrice André ${ }^{2}$ and Claire Elfakir ${ }^{1}$ \\ 1 ICOA, Université d'Orléans, CNRS, UMR 7311, Orléans F-45067, France; \\ E-Mails: saida.el-abdellaoui@espci.fr (S.E.A.); laetitia.fougere@univ-orleans.fr (L.F.); \\ ajbtoribio@msn.com (A.T.); claire.elfakir@univ-orleans.fr (C.E.) \\ 2 LVMH Recherche Parfums et Cosmétiques, Saint Jean de Braye 45800, France; \\ E-Mails: irenimel@research.lvmh-pc.com (I.K.-R.); patrice.andre0@orange.fr (P.A.) \\ 3 GLYcoDIAG, Université d'Orléans, Orléans Cedex 2 45067, France; \\ E-Mails: cancellieri@glycodiag.com (P.C.); landemarre@glycodiag.com (L.L.)
}

* Author to whom correspondence should be addressed; E-Mail: emilie.destandau@univ-orleans.fr; Tel.: +33-2384-17074; Fax: +33-2384-17281.

Received: 25 September 2013; in revised form: 16 December 2013 / Accepted: 23 December 2013 / Published: 2 January 2014

\begin{abstract}
To develop a new antioxidant, antibacterial and natural cosmetic ingredient without cytotoxicity to skin cells, bioactive molecules contained in Kalanchoe pinnata leaf methanolic extract were targeted using semi-preparative HPLC fractionation linked to biological activity tests. Chromatographic effluent was collected at the column outlet into a 96 deep-well microplate, filling successively all the wells. After freeze-drying, the microplate was ready to use for different biological tests such as antimicrobial activity on microorganisms, skin cell viability and antioxidant activity on human keratinocyte cells. The injection of only $2.64 \mathrm{mg}$ of crude extract into the HPLC system reveals a good correlation between the chromatographic peaks and the different biological activities. One fraction is mainly of interest since good antibacterial and antioxidant activities without cytotoxicity are observed. The analysis of this fraction using mass spectrometry allows the identification of glycoside derivatives of quercetin, isorhamnetin and kaempferol. Thus, a correlation between biological activity and the presence of these flavonoids is obtained. This screening method allows a rapid fractionation associated with a biological activity evaluation and a first molecular identification, saving time by limiting sample treatments and solvent consumption.
\end{abstract}


Keywords: Bryophyllum pinnatum; semi-preparative HPLC fractionation; microorganisms; human keratinocytes; cytotoxicity; mass spectrometry

\section{Introduction}

As part of a collaborative project aimed to isolate new natural ingredients for cosmetic usage that possess anti-ageing properties and are able to replace synthetic preservatives in formulations, about 50 plant organs from Guyana were selected. These plants were chosen according to the local culture and their traditional use.

The conventional approach for screening biological activities of plant involves an extraction step followed by a fractionation step often using liquid chromatography on open column consuming hundreds of milligrams of raw material, a large amount of solvent and requiring a long sample treatment to be suitable for biological tests. However, in this project, we developed an easier, faster and miniaturized screening method to be able to evaluate numerous extracts using small amounts of plant material and solvent. Hence, a semi-preparative HPLC-based activity profiling method was chosen [1]. Thus, the fractionation at the milligram scale of a crude plant extract on semi-preparative HPLC column can be followed by biological activity tests. To avoid missing minor compounds with potential high activity, the chromatographic effluent is continuously collected at the column end onto a 96 deep-well plate ready-to-use for biological tests later. As plant extracts are dedicated to anti-ageing and preservative formulations, antimicrobial activity on microorganisms, skin cell viability and antioxidant protection of normal human keratinocytes against reactive oxygen species (ROS) were assessed using miniaturized protocols. Correlation between fractionation chromatogram and active wells on microplate could indicate which molecules participate in crude extract activity.

Therefore, to estimate the suitability of this method to bring out antibacterial and antioxidant activities and to screen bioactive molecules in plant extract, Kalanchoe pinnata leaf extract was selected among the 50 plant organs as a plant model to validate our protocol before applying it to other plant extracts. Kalanchoe pinnata (Lam.) Pers. (syn. Bryophyllum pinnatum) (family Crassulaceae) is a perennial plant which grows in Africa and Asia. This popular plant, used medicinally in many regions of the world, is applied externally or taken internally for all types of pains and inflammation [2]. The K. pinnata leaves are traditionally used as anti-inflammatory and antiseptic for treating coughs, sores, wounds and cuts [3-5]. Juice of the fresh leaves is used very effectively for the treatment of jaundice in folk medicines of the Bundelkhand region of India [6]. The hematoprotective [6], immunosuppressive [7], antiulcer [8], anti-inflammatory [9,10] and antimicrobial activity [4,11,12] of the leaf extract have also been reported. Moreover, several pharmacological studies have revealed its anticancer [13] and antileishmanial properties [14-16]. These beneficial effects are attributed to several classes of molecules identified in K. pinnata extracts. Anticancer properties are correlated to the presence of bufadienolides and bryophyllium derivatives [13]. Previous studies have shown the importance of flavonoids for antioxidant [17], antimicrobial [4,12,18] and antileishmanial activities [15,16]. Some phenolic compounds [19] and fatty acids [7] have also been identified in K. pinnata. 
This paper describes a fast and easy fractionation method leading to immediate isolation of antibacterial, antioxidant and, moreover, non-cytotoxic compounds. Screening methodology is miniaturized reducing raw material and solvent consumption. The direct correlation between fractionation and biological activity tests limits sample preparation and improves the rapid highlighting of fraction of interest. The identification of the bioactive compounds contained in the fraction of interest is finally performed using mass spectrometry. Hence, with a demonstrated biological activity and a known molecular content, fractions of interest can be selected to be integrated into cosmetic formulations as preservative and anti-ageing ingredients.

\section{Experimental Section}

\subsection{Reagents}

Ultra pure water was purified (conductivity $<18 \mathrm{M} \Omega$ ) from distilled water using an Elgastat UHQ II system (Elga, Antony, France). Organic solvents such as acetonitrile, ethanol and methanol, formic acid were all HPLC grade and purchased from SDS Carlo Erba (Val de Reuil, France). Keratinocyte Serum-Free Medium (K-SFM) was obtained from Life Technologies (Saint Aubin, France). Cell culture microplates were purchased from Greiner (Courtaboeuf, France). Cell Proliferation kit II (XTT test) was purchased from Roche Diagnostics (Meylan, France). Protein determination kit (BCA assay) was purchased from Sigma (St. Louis, MO, USA) and DCFH-DA probes were obtained from Sigma (St. Louis, MO, USA).

\subsection{Kalanchoe pinnata Extract}

The leaves of $K$. pinnata (Lam.) Pers. (syn. Bryophyllum pinnatum; family Crassulaceae) were collected in September 2006, in the French Guyana region of Kaw. The GPS coordinates were N04 $33^{\prime} 200^{\prime \prime}$, W52 $12^{\prime} 685^{\prime \prime} 254 \mathrm{mt}$. The dried leaves were crushed into a basic grinder and then submitted to pressurized liquid extraction using an ASE 100 from Dionex (Voisins le Bretonneux, France). Extraction conditions were methanol as extraction solvent, temperature $40{ }^{\circ} \mathrm{C}$, static time 5 min, 3 cycles, flush $60 \%$, purge time $100 \mathrm{~s}$ [12]. The extraction yield, defined as the dried crude extract mass on the dried raw material mass, was $8 \%$.

\subsection{Semi-Preparative HPLC Fractionation}

The fractionation method, presented on Scheme 1, was performed on an Agilent 1100 (Palo Alto, CA, USA) system equipped with a binary pump, an oven and a UV detector. Separation was carried out using a Hypersil H5C18 $(250 \times 10 \mathrm{~mm}, 5 \mu \mathrm{m})$ column with a water/methanol gradient elution: from 0-5 min: 5\% MeOH, from 5-30 min: 5\%-100\% $\mathrm{MeOH}$, and from 30-40 min: 100\% $\mathrm{MeOH}$. Flow rate was $1 \mathrm{~mL} \mathrm{~min}^{-1}$ and separation was performed at room temperature. Injected crude extract solution was prepared at $132 \mathrm{mg} \mathrm{mL}^{-1}$. To evaluate the sensibility of the biological tests and the lowest amount of raw material needed to perform them, three amounts of crude extract were injected onto the semi-preparative HPLC column: $0.66 \mathrm{mg}(5 \mu \mathrm{L}$ injected), $1.32 \mathrm{mg}(10 \mu \mathrm{L}$ injected) and $2.64 \mathrm{mg}$ (20 $\mu \mathrm{L}$ injected). 
Scheme 1. HPLC semi-preparative fractionation method of Kalanchoe pinnata leaf methanolic extract. HPLC effluent was monitored at $254 \mathrm{~nm}$ and directly collected in a 96 deep-well plate ready to use for biological activity tests.

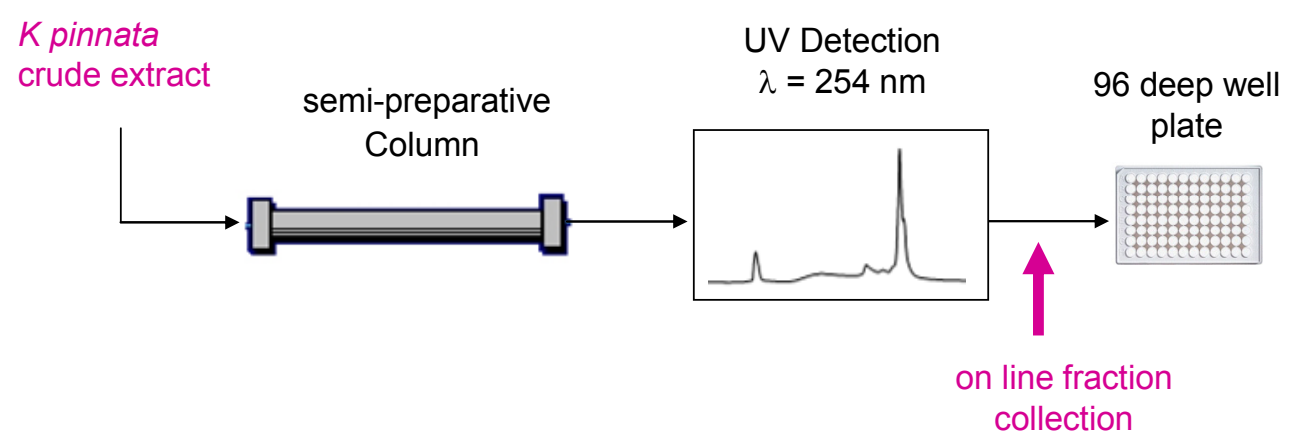

During the HPLC separation process, the effluent was monitored by UV at $254 \mathrm{~nm}$ and continuously collected in a 96 deep-well plate. Each well was filled for 1.6 min corresponding to an effluent volume of $1.6 \mathrm{~mL}$. As each HPLC separation was realized with a run time of $40 \mathrm{~min}$, each injected crude extract amount led to 25 fractions filling only 25 wells. Hence, effluent collected for the three injected amounts of crude extract $(0.66 \mathrm{mg}, 1.32 \mathrm{mg}$ and $2.64 \mathrm{mg}$ ) could be deposited onto the same 96 deep-well plate as well as the three crude extract samples with a view to establish the relationship between extract concentration and biological activity.

Once the fractionation was made, the plates were freeze-dried overnight and were ready to use for later biological studies. The plates used for mass spectrometry analysis did not need the evaporation step.

\subsection{Mass Spectrometry}

The chromatographic effluent collected in the 96 deep-well plate was directly analyzed without any preparation or concentration by Flow Injection Analysis in Mass Spectrometry (FIA-MS) with an AB Sciex API 3000 triple quadruple mass spectrometer equipped with a TurboIonSpray source (Forster City, CA, USA). Twenty microliter of sample contained in each well was injected in a liquid effluent composed of water/methanol (5/95) acidified with $0.1 \%$ formic acid at a flow rate of $0.3 \mathrm{~mL} \mathrm{~min}$ and $^{-1}$ introduced in the electrospray ionization source. The mass spectrometer operated in negative ionization mode. Nitrogen was used as curtain and nebulizer gas. The spectrometer parameters were set as follow: nebulizer gas flow rate, $\mathrm{NEB}=8\left(1.2 \mathrm{~L} \mathrm{~min}^{-1}\right)$; curtain gas flow rate, $\mathrm{CUR}=8\left(1.2 \mathrm{~L} \mathrm{~min}{ }^{-1}\right)$; ion spray voltage, IS $=-4200 \mathrm{~V}$; declustering potential, $\mathrm{DP}=-100 \mathrm{~V}$; focusing potential, $\mathrm{FP}=-400 \mathrm{~V}$; entrance potential, EP $=-10 \mathrm{~V}$. Mass spectra were recorded in full scan mode in the range 100-1000 u with a step size of $0.5 \mathrm{u}$, in order to detect the maximum of ions and attempt identification of compounds. For MS/MS experiment, nitrogen was used as collision gas and collision energy was set at $30 \mathrm{eV}$. All results were acquired with the Analyst software version 1.4.2 (Sciex Applied Biosystems, Forster City, CA, USA).

\subsection{Antibacterial Assay}

A micro-method, derived from the preservative efficacy testing described by Orth et al. [20] and allowing the screening of a large number of samples was developed. The microorganisms used include 
all the strains recommended in the current regulatory method for cosmetic preservative efficacy testing (EN ISO11930): Pseudomonas aeruginosa (Pa) ATCC 9027, Staphylococcus aureus (Sa) ATCC 6538, Escherichia coli (Ec) ATCC 8739, Candida albicans (Ca) ATCC 10231, Aspergillus brasiliensis (Ab) ATCC 16404. These microorganisms were grown $24-48 \mathrm{~h}$ at $35^{\circ} \mathrm{C}$ on tryptone soya broth (bacteria), YM broth (Candida albicans) or at $25^{\circ} \mathrm{C}$ on Sabouraud broth (Aspergillus brasiliensis) before preparation of a pure saline inoculum containing between $10^{6}$ and $10^{7}$ (fungus and yeast) or between $10^{7}$ and $10^{8}$ (bacteria) colony formation unit (CFU) $\mathrm{mL}^{-1}$. Each fraction of the deep well plate was first dissolved in DMSO, separated in five deep-wells (one for each tested microorganism) and then contaminated with $10 \mu \mathrm{L}$ of inoculum of each microorganism and incubated at $22{ }^{\circ} \mathrm{C}$. Phenonip $0.5 \%$ was used as reference standard and was subjected to the same treatment as fractions. Microbial counts were done at days 7, 14, 21 and 28 days for each fraction.

\subsection{Cell Culture}

Normal Human Epidermis Keratinocytes (NHEK) were used for cytotoxic and antioxidant activities. They were obtained from an abdominal surgery and provided by LVMH Recherche. Cells were cultivated in Keratinocyte Serum-Free Medium (K-SFM) from Gibco with $50 \mu \mathrm{g} \mathrm{mL}{ }^{-1}$ bovine pituitary extract, $0.1 \mathrm{ng} \mathrm{mL}^{-1}$ epidermal growth factor, and $0.4 \mathrm{mM} \mathrm{CaCl}_{2}$. For all experiments, cells used were on their 5 th passage. They were seeded at a density of $5 \times 10^{4} \mathrm{cells} \mathrm{cm}^{-2}$ in K-SFM medium and incubated at $37{ }^{\circ} \mathrm{C}$ in $5 \% \mathrm{CO}_{2}$ for $24 \mathrm{~h}$ to allow adhesion. Cell confluence at the time of experiment was approximately $80 \%$.

\subsection{Skin Cell Viability Test}

The test was assessed using a modified XTT assay [21]. The method is based on the ability of metabolically active cells to convert yellow tetrazolium salt XTT into orange formazan dye. As this conversion occurs only in live cells, cytotoxicity of various chemical substances can be determined in this way. The originating formazan dye is soluble in water and can be directly quantified using any classical spectrophotometer. Briefly, NHEK were seeded in a 96-well plate and treated with $K$. pinnata extracts or fractions or vehicle (DMSO used as reference standard) for $20 \mathrm{~h}$. Then, the medium was discarded and $200 \mu \mathrm{L}$ of XTT solution (according to the protocol proposed in the "cell proliferation kit") was added to each well and further incubated for $4 \mathrm{~h}$ at $37{ }^{\circ} \mathrm{C}$. The absorbance of each well was measured at $450 \mathrm{~nm}$ with a microplate reader (Tecan, France).

\subsection{Antioxidant Assay on Normal Human Epidermis Keratinocytes}

Radical scavenging activity was done on the cell layer using the DCFH-CA probe. The DCFH-DA probe diffuses across the cell membrane and is then hydrolyzed by intracellular esterase to free DCFH. When Reactive Oxygen Species (ROS) are present, the free DCFH probe is converted into a highly fluorescent compound 2',7'-dichlorofluorescein (DCF) [22]. NHEK, grown in a supplemented K-SFM, were seeded in 96-well black microplate $\left(75,000\right.$ cells $\mathrm{mL}^{-1}$ ) purchased from Greiner (Courtaboeuf, France). After $24 \mathrm{~h}$ of incubation, the medium was replaced by K-SFM containing $K$. pinnata extracts or fractions. After sample treatment, the medium was discarded, the cells were rinsed with PBS and 
incubated with $50 \mu \mathrm{M}$ DCFH-DA in the medium at $37{ }^{\circ} \mathrm{C}$ and agitated for $45 \mathrm{~min}$. Afterwards DCFH-DA was removed, the cells were washed with PBS and incubated with PBS containing $100 \mu \mathrm{M} \mathrm{H}_{2} \mathrm{O}_{2}$ as free radical generator for $20 \mathrm{~min}$. To verify if the tested molecules have no pro-oxidant activity, cells were also treated with tested $K$. pinnata extracts and fractions without $\mathrm{H}_{2} \mathrm{O}_{2}$ induction. DCFH-DA fluorescence intensity produced by loaded cells was measured with Spectrafluor Plus microplate fluorescence reader (TECAN, France) at room temperature. The excitation filter was set at $485 \mathrm{~nm}$ and the emission filter was set at $535 \mathrm{~nm}$. The fluorescence intensity was directly proportional to the concentration of free radical compounds. The percentage of protection of the antioxidant was calculated by comparing the results of the cells treated with the antioxidant to the untreated control (solvent control) cells.

\section{Results and Discussion}

\subsection{Semi-Preparative HPLC Fractionation}

Fractionation of $K$. pinnata leaf crude extract was carried out with reversed liquid chromatography on a semi-preparative column. The goal was not to achieve a complete separation of all extracted compounds but to easily bring out the most active fractions that may contain several interesting molecules with potential industrial applications. Figure 1 shows the chromatographic profiles recorded for the three injected amounts $0.66 \mathrm{mg}, 1.32 \mathrm{mg}$ and $2.64 \mathrm{mg}$. Similar profiles are obtained with an increase of peak intensity when the injected amount increases. A small peak at $8 \mathrm{~min}$, a wide undefined band of 13-20 min, three small peaks between 20 and $25 \mathrm{~min}$ and, finally, a high intensity peak at 26 min are observed. After this, signal returns to baseline until the end of the elution gradient program.

Figure 1. Chromatographic profiles of Kalanchoe pinnata leaf methanolic crude extract. Hypersil H5C18 $(250 \times 10 \mathrm{~mm}, 5 \mu \mathrm{m})$ column with a water/methanol gradient elution: from 0-5 min: 5\% MeOH, 5-30 min: 5\%-100\% MeOH, and 30-40 min: 100\% $\mathrm{MeOH}$. Flow rate $1 \mathrm{~mL} \mathrm{~min}^{-1}$, room temperature, detection $\lambda=254 \mathrm{~nm}$, injection of (A) $0.66 \mathrm{mg}$ (B) $1.32 \mathrm{mg}$ (C) $2.64 \mathrm{mg}$.

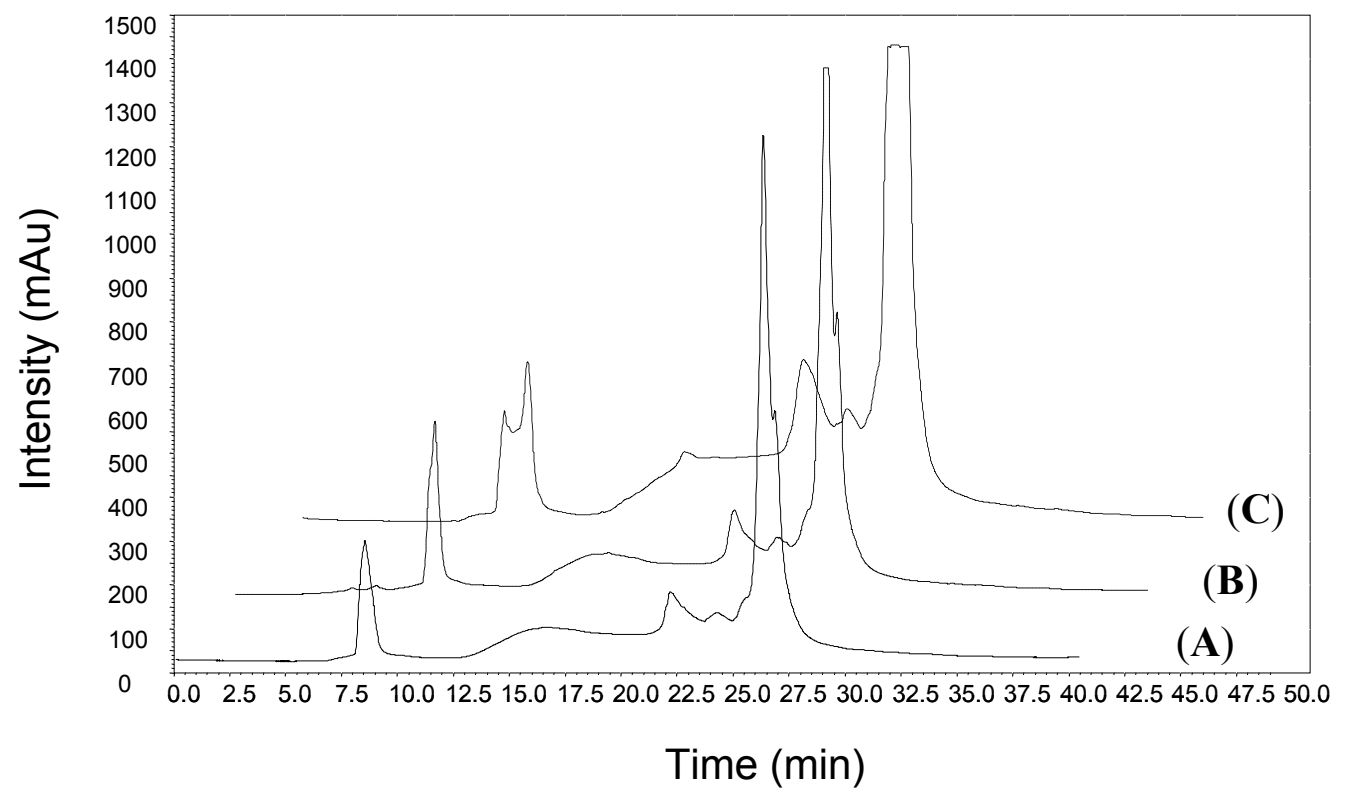


In order to retrieve the entire contents of the crude extract, the chromatographic effluent was collected at the column outlet in different wells and all wells were evaluated using biological activity tests. Hence, the biological activity of separated chromatographic peak could be performed. To be sure that all compounds were evaluated, base-line signal parts of the chromatogram were also collected since some minor compounds may be under the UV detection limit and some molecules that do not absorb at $254 \mathrm{~nm}$ may have important activity. Fractions containing only mobile phase could also be considered as negative control to validate the biological response.

Unlike the classical methods where samples need to be evaporated and diluted at different concentrations during the biological assay to establish dose-response curves, here the direct HPLC injection of different crude extract concentrations leads to this dose-response information avoiding preparation of dilution range. Hence, it may be possible to detect an evolution of the activity with both extract and fraction concentration and to evaluate the lower amount of raw material required to obtain a biological response.

Moreover, as all the fractions are obtained directly from the crude extract injection, the concentration of the different molecules for their biological evaluation is not identical but is proportional to their real concentration in the crude extract. Hence, the activity observed for the fractions reflects the real contribution of their molecules to the entire crude extract activity. Thus, if a compound is identified as active it means that both its own activity and its concentration in the extract were sufficient to allow the activity detection.

In order to control the method repeatability and the homogeneity of the results obtained from the different biological tests, three plates (78 samples each) were realized. The first one was used for antibacterial assays, the next one for cytotoxicity evaluation and antioxidant test and the last one for mass spectrometry. The fractionation was carried out with a good repeatability suggesting a similar repartition of molecules in each deep well plate and which allows an easy comparison of the different tests.

\subsection{Antibacterial Assay}

The antibacterial effect was measured on Escherichia coli (Ec), Staphylococcus aureus (Sa), Pseudomonas aeruginosa (Pa), Candida albicans (Ca) and Aspergillus brasiliensis (Ab). Crude extract activity was tested at the three injected concentrations of 264, 132 and $66 \mu \mathrm{g} \mathrm{mL}^{-1}$, respectively. Results gathered in Table 1 show a bacterial inhibition of Sa and $\mathrm{Ca}$ at 28 days with the lowest injected concentration $\left(66 \mu \mathrm{g} \mathrm{mL}^{-1}\right)$. This inhibition increases when a higher extract concentration is tested $\left(132 \mu \mathrm{g} \mathrm{mL}^{-1}\right)$. The inhibition kinetic is also improved when extract concentration reached $\left(264 \mu \mathrm{g} \mathrm{mL}^{-1}\right)$ since bacterial population growth of the five strains is inhibited in 21 days. These results afford a better sensibility than those described by Akinpelu for a $60 \%$ methanolic extract by agar-well diffusion method [4] and seem to be in accordance with those proposed by Tatsimo et al. that observed MIC (Minimum Inhibitory Concentration) ranging from $32-512 \mu \mathrm{g} \mathrm{mL}^{-1}$ for methanolic extract [18].

Concerning fractions, Figure 2 shows, as an example, the correlation between HPLC profile and antibacterial activity on Escherichia coli strains at 28 days of incubation for the three concentration levels of fractionated K. pinnata extract. 
Table 1. Antibacterial activity of Kalanchoe pinnata leaf methanolic crude extract (CE) and fractions obtained by semi-preparative HPLC on Escherichia coli (Ec), Staphylococcus aureus (Sa), Pseudomonas aeruginosa (Pa), Candida albicans (Ca) and Aspergillus brasiliensis (Ab).

\begin{tabular}{|c|c|c|c|c|c|}
\hline Samples & Ec & Sa & $\mathbf{P a}$ & $\mathbf{C a}$ & $\mathbf{A b}$ \\
\hline $\mathrm{CE} 66 \mu \mathrm{g} \mathrm{mL} L^{-1}$ & - & ++ & - & ++ & - \\
\hline $\mathrm{CE} 132 \mu \mathrm{g} \mathrm{mL}^{-1}$ & + & + & - & ++ & - \\
\hline $\mathrm{CE} 264 \mu \mathrm{g} \mathrm{mL}^{-1}$ & + & + & $+/-$ & + & + \\
\hline Fraction 1 & + & + & - & $+/-$ & - \\
\hline Fraction 2 & + & + & - & $+/-$ & - \\
\hline Fraction 3 & ++ & + & $+/-$ & $+/-$ & - \\
\hline Fraction 4 & - & + & $+/-$ & $+/-$ & - \\
\hline Fraction 5 & - & + & - & $+/-$ & - \\
\hline Fraction 6 & $+/-$ & + & - & $+/-$ & - \\
\hline Fraction 7 & - & + & - & ++ & - \\
\hline Fraction 8 & - & + & + & ++ & - \\
\hline Fraction 9 & + & + & + & ++ & - \\
\hline Fraction 10 & + & + & - & $+/-$ & - \\
\hline Fraction 11 & - & ++ & - & $+/-$ & - \\
\hline Fraction 12 & - & ++ & - & $+/-$ & - \\
\hline Fraction 13 & + & ++ & - & $+/-$ & - \\
\hline Fraction 14 & - & ++ & - & ++ & - \\
\hline Fraction 15 & ++ & - & - & ++ & - \\
\hline Fraction 16 & ++ & + & + & ++ & - \\
\hline Fraction 17 & $+1-$ & + & + & $+/-$ & - \\
\hline Fraction 18 & + & + & + & $+/-$ & - \\
\hline Fraction 19 & - & + & - & $+/-$ & - \\
\hline Fraction 20 & - & + & - & ++ & - \\
\hline Fraction 21 & - & + & - & ++ & - \\
\hline Fraction 22 & - & + & - & ++ & - \\
\hline Fraction 23 & + & ++ & - & $+/-$ & - \\
\hline Fraction 24 & + & + & - & $+/-$ & - \\
\hline Fraction 25 & + & + & + & ++ & - \\
\hline
\end{tabular}

$(++)$ active sample at low concentration (0.66 mg injected); $(+)$ active sample at medium (1.32 mg) or high (2.64 mg) concentration; (+/-) sample that inhibits only a part of bacterial population at high concentration; $(-)$ non active sample. In gray are the most active fractions.

It appears that molecules eluted through the first and the third chromatographic peaks inhibit bacterial growth from the $0.66 \mathrm{mg}$ injected, while molecules corresponding to the less intense second chromatographic peak show activity only for the injection of $2.64 \mathrm{mg}$. The inhibition of bacterial growth is crude extract and fraction concentration dependent. When compound concentration increases, inhibition of bacterial growth is more efficient. Injection of $0.66 \mathrm{mg}$ of crude extract appears sufficient to reveal the antibacterial activity of the most concentrated or the most active compounds present in this extract. At this concentration, empty chromatographic zones do not show a valuable inhibition of 
bacterial growth indicating that potential undetected compounds do not possess high antibacterial activity and that the chromatographic mobile phase itself did not induce antibacterial response.

Figure 2. Correlation between chromatographic profile of Kalanchoe pinnata leaf methanolic extract and antibacterial activity against Escherichia coli strain.

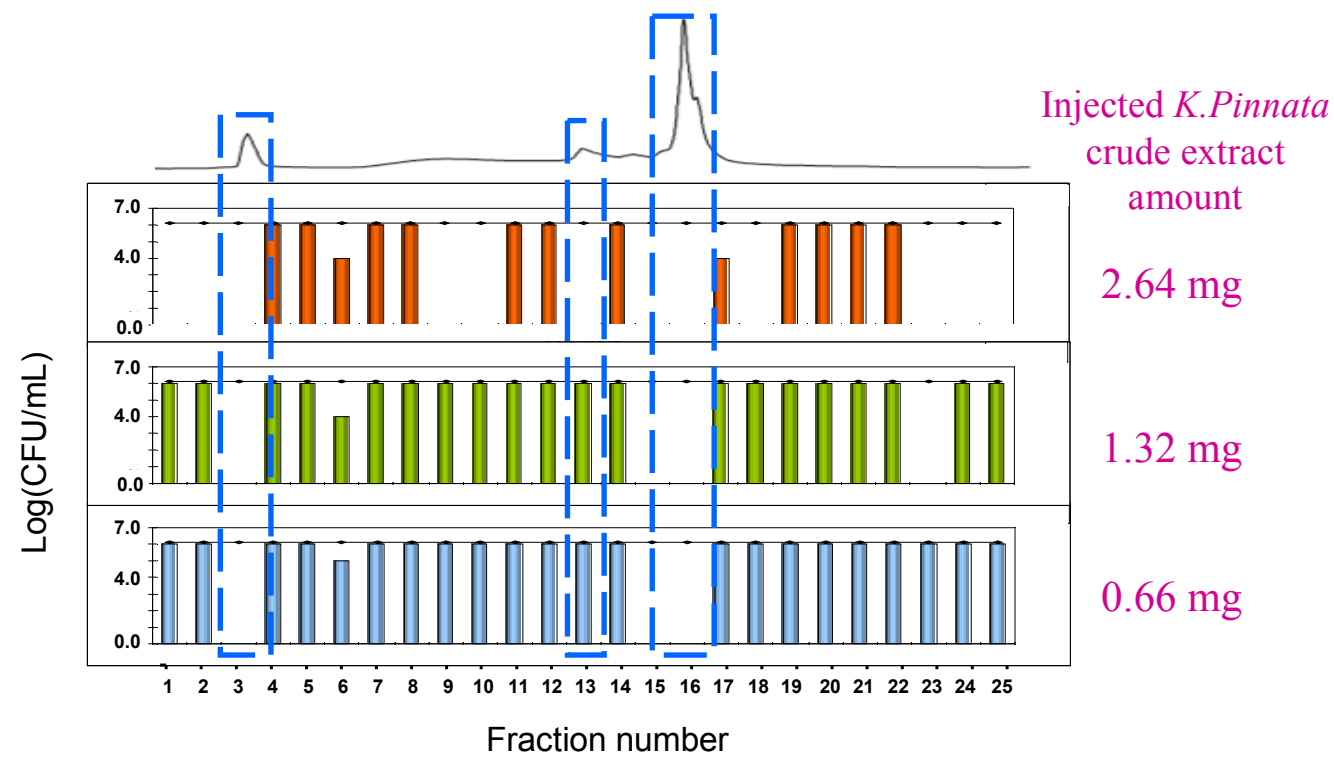

Results obtained for crude extract and fractions on the five strains are gathered in Table 1. Sa is the most sensitive strain with an inhibition of growth for all fractions, while Ab's response to the various fractions is not affected and only a low activity is observed for the most concentrated crude extract. $\mathrm{Pa}$ and $\mathrm{Ca}$ growth are inhibited by some fractions. In brief, the cumulating behavior of the five strains contaminated with $K$. pinnata fractions is as follows: fraction 3 corresponding to the first chromatographic peak, fraction 9, fractions $15,16,17$ corresponding to the third chromatographic peak and fraction 25 at the end of the chromatogram are the fractions with the highest antibacterial activity.

\subsection{Viability Test}

The cytotoxicity of samples was assessed using a modified XTT assay. Crude methanolic extract of $K$. pinnata leaves presents a high cytotoxic effect [12]. However, it is not usable in this form for cosmetic applications. If the cytotoxic molecules are not the antibacterial ones, the extract fractionation may lead to a fraction with an interesting antibacterial effect but without cytotoxicity. Figure 3 presents for the injected crude extract amount of $2.64 \mathrm{mg}$ the not-cytotoxic concentration of each collected fraction. Chromatographic peaks 1 and 2 show a low not-cytotoxic concentration $\left(<10 \mu \mathrm{g} \mathrm{mL}{ }^{-1}\right)$, indicating the cytotoxicity of molecules eluted in these peaks. Whereas the third peak seems to be more interesting since the not-cytotoxic concentration is higher $\left(50 \mu \mathrm{g} \mathrm{mL}^{-1}\right)$ at the same level as chromatographic empty zones. The recommended not-cytotoxic concentration for a safe sample use is in the range $50-12.5 \mu \mathrm{g} \mathrm{mL}^{-1}$. Considering also the potentially higher concentration of these molecules, they could be considered as not-cytotoxic molecules, incorporable in cosmetic formulations. Lower injected concentrations ( 0.66 and $1.32 \mathrm{mg})$ did not present cytotoxic effect. 
Figure 3. Correlation between chromatographic profile of Kalanchoe pinnata leaf methanolic extract and cytotoxicity and antioxidant DCHH-DA activity.

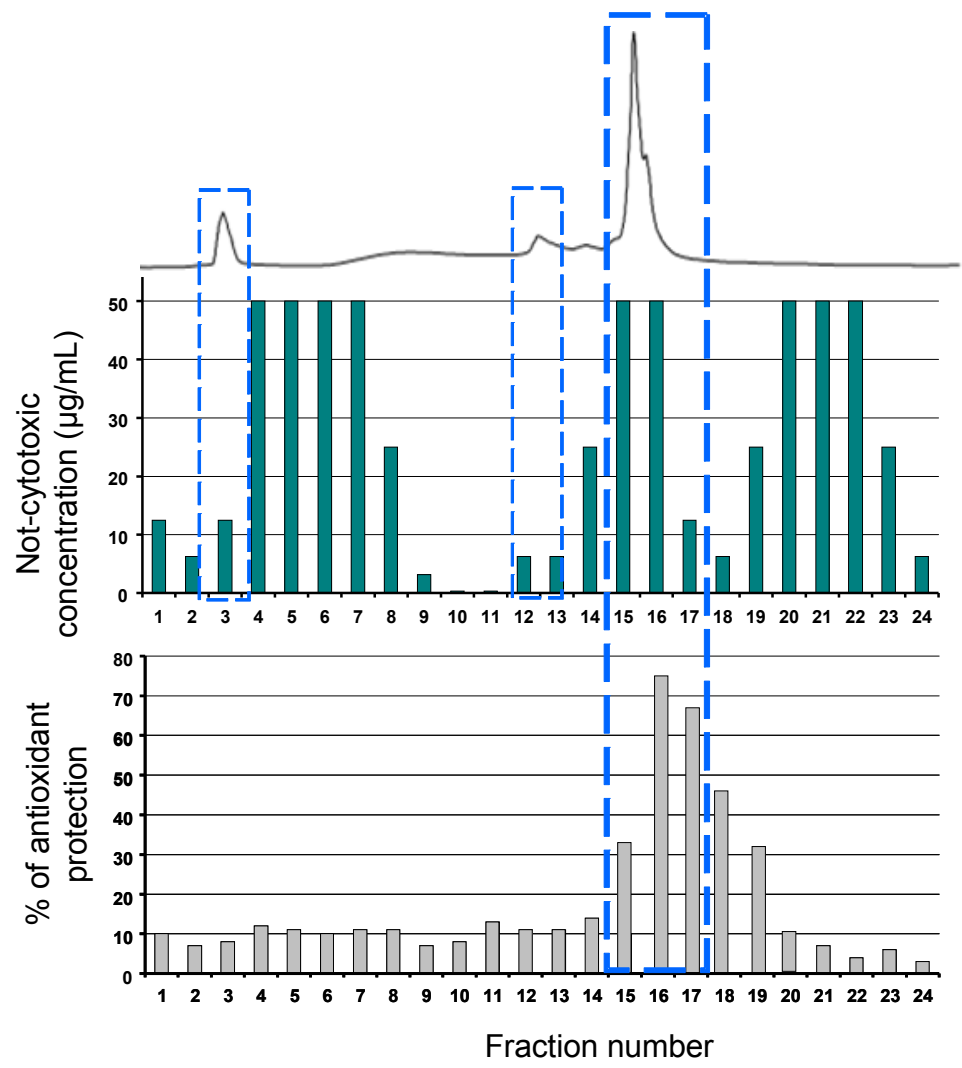

\subsection{Antioxidant Assay on Normal Human Epidermis Keratinocytes}

Previous antioxidant activity of $K$. pinnata extracts has been evaluated mainly with in vitro assays as 2,2'-diphenyl-1-picrylhydrazyl (DPPH) free radical method [17,18]. In this work, to consider the use of $K$. pinnata in cosmetics, antioxidant activity was evaluated on Normal Human Epidermis Keratinocytes with the DCFH-DA method. Indeed, keratinocyte cells - major cells of epidermis - play an important role in the skin ageing and particularly in the ageing induced by oxidative stress and chronic inflammation [23,24]. Oxidative stress was induced by incubating the keratinocyte cells with $100 \mu \mathrm{M} \mathrm{H}_{2} \mathrm{O}_{2}$, a high level of reactive oxygen species (ROS) were then produced. Protection against ROS when $K$. pinnata extracted molecules were present was measured. Compared to non-treated cells, ROS production induced by $\mathrm{H}_{2} \mathrm{O}_{2}$ was significantly decreased in the case of keratinocyte cells treated with $K$. pinnata extract or fractions. Protection occurred in a dose-dependent manner since it was efficient only with injection of $2.64 \mathrm{mg}$ of crude extract. Lower injected amounts were not sufficient. Figure 3 depicts the correlation between HPLC chromatogram and the cell protection against ROS. A good correlation is observed. The high third peak allows a good protection of keratinocytes with more than $70 \%$ of antioxidant protection while the first two peaks do not show antioxidant protection.

Molecules eluted under the third chromatographic peak and corresponding mainly to fractions 15 and 16 on the deep-well plate present good advantages; they exhibit good antibacterial and antioxidant activities and a low cytotoxicity. Compared to the crude extract, these simplified fractions allow a concentration of antibacterial and antioxidant molecules and an elimination of cytotoxic compounds; 
therefore, they could be considered as an active and safe potential new natural ingredient for cosmetics. In order to evaluate the composition of these fractions, mass spectrometry analyses were performed directly on the HPLC effluent collected in the corresponding deep-well plate.

\subsection{Mass Spectrometry}

Content of fractions 15 and 16 in which the interesting molecules were eluted was analyzed by Flow Injection Analysis with an Electrospray ion source. In order to detect all ions without any pre-selection, MS analyses were performed in full scan mode from 100-1000 u. Compared to positive ionization mode, the negative one showed higher signal sensitivity and in-source fragmentation allowing to attempt compound identification in full scan analysis mode. The fragmentation pattern supposed from the full scan was then confirmed by MS/MS experiment with fragmentation of the deprotonated molecule ions $[\mathrm{M}-\mathrm{H}]^{-}$.

Figure 4. Full scan mass spectrum of fractions 15 and 16 recorded with ESI in negative ionization mode.
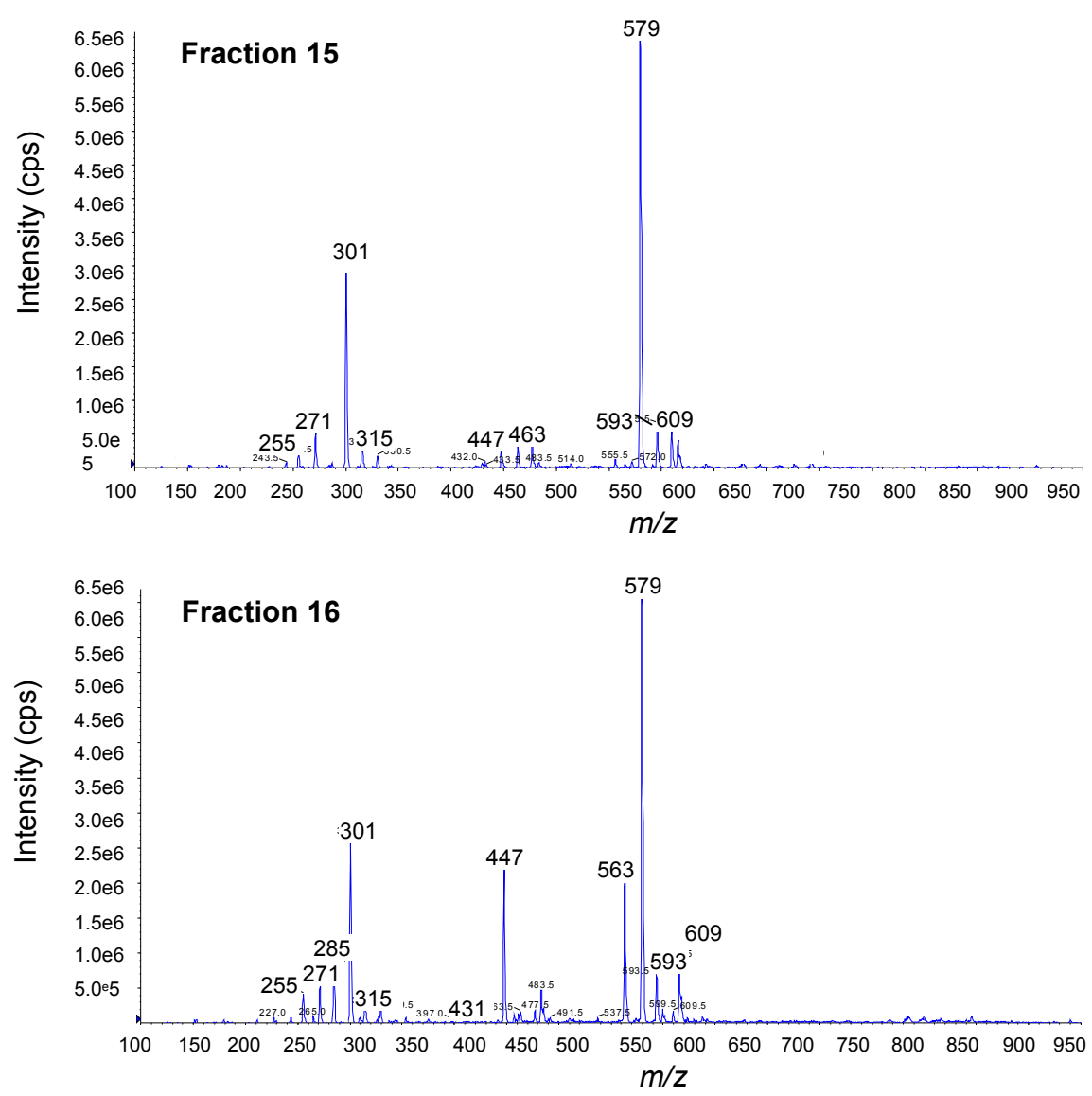

UV spectrum (not shown) recorded during the MS experiment shows absorbance maxima at 250-260 nm for band II and at 350-360 nm for band I characteristic to flavonoid compounds. Moreover, Figure 4 presents the full scan MS spectrum of fraction 15. One main molecule with a $[\mathrm{M}-\mathrm{H}]^{-}$at $\mathrm{m} / \mathrm{z} 579$ and fragment ions at $\mathrm{m} / \mathrm{z} 447$ and 301 could be observed indicating the loss of 132 and $146 \mathrm{u}$ corresponding to the loss of pentose and deoxyhexose, respectively. The last fragment ion $[\mathrm{M}-\mathrm{gly}-\mathrm{H}]^{-}$at $\mathrm{m} / \mathrm{z} 301$ is characteristic to the quercetin genin, that could be confirmed also with 
the detection of ions at $m / z 271$ and 255 due to Retro Diels Alder (RDA) rearrangements in quercetin genin [25]. MS/MS analysis of the $[\mathrm{M}-\mathrm{H}]^{-}$confirmed this fragmentation pattern. Hence, this main molecule could be identified as quercetin-3-O- $\alpha$-arabinopyranosyl (1-2) $\alpha$-rhamnopyranoside that has been already described as the main compound in $K$. pinnata leaf methanolic extract and has been suggested as phytochemical marker for this plant species [15]. Other less concentrated molecules could be detected also. $[\mathrm{M}-\mathrm{H}]^{-}$at $\mathrm{m} / z 609$ gave after fragmentation ion fragments at $\mathrm{m} / \mathrm{z} 463$ and 301 indicating the loss of 146 and $162 \mathrm{u}$ corresponding to the loss of deoxyhexose and hexose and the presence of a quercetin genin. This molecule could be identified as rutin. Finally, $[\mathrm{M}-\mathrm{H}]^{-}$at $m / z 593$ that gave after fragmentation ion at $m / z 315$ indicating the loss of $278 \mathrm{u}$ that could be due to the loss of 132 and $146 \mathrm{u}$ due to pentose and dehoxyhexose units like rhamnose and arabinose. Ion Fragment $[\mathrm{M}-\text { gly }-\mathrm{H}]^{-}$at $\mathrm{m} / \mathrm{z} 315$ could be characteristic to an isorhamnetin (or isomer) genin.

According to the shape of the chromatographic peak (Figure 1), it is obvious that other molecules should be eluted in fraction 16. MS spectrum of fraction 16 presented in Figure 4 shows effectively an evolution in the proportion of the compounds previously found in fraction 15. Moreover, another $[\mathrm{M}-\mathrm{H}]^{-}$at $m / z 563$ leading to fragment ions at $\mathrm{m} / \mathrm{z} 431$ and 285 with loss of 132 and $146 \mathrm{u}$ indicates the presence of Kaempferol-arabinose-rhamnose. Kaempferol derivatives have been previously described in K. pinnata [18]. Later elution of kaempferol derivatives is not surprising since kaempferol possess less hydroxyl group than quercetin. Briefly, glycoside (mainly arabinose and rhamnose) derivatives of quercetin, isorhamnetin and kaempferol have been detected in these fractions and appear to be responsible for the antibacterial and antioxidant activity of $K$. pinnata leaf methanolic extract.

Well known cytotoxic bufadienolide compounds of $K$. pinnata potentially extracted were not detected in these fractions and seemed to be well separated from flavonoid compounds. This could explain the low cytotoxicity of the fractions compared to the crude extract one.

\section{Conclusions}

The developed semi-preparative HPLC fractionation linked to biological activity tests allowed bringing out antioxidant, antibacterial and non-cytotoxic molecules from Kalanchoe pinnata methanolic crude extract, saving time, sample treatment, solvent and raw material consumption. Only some milligrams of crude extract were required to assess biological tests and mass spectrometry analysis, and sample preparation was limited to extraction and evaporation steps. It was possible to obtain rapidly simplified fractions of crude extract, to test their biological activity and to correlate this activity to the molecules contained in these fractions. Moreover, molecules were tested in the fractions at their relative concentration in the crude extract that indicates their real participation in the crude extract activity.

This methodology highlighted an interesting antibacterial and antioxidant fraction that inhibits the growth of Ec, Sa, Pa and $\mathrm{Ca}$ and that protects more than $70 \%$ of normal human keratinocyte cells against ROS. Keratinocyte cells - major cells of epidermis - play an important role in skin ageing and, particularly, in the ageing induced by oxidative stress and chronic inflammation [23,24]. Oxidative stress is the consequence of our environment (UV radiation) and our life style (smoke cigarette, for example). UV radiation is known to increase skin inflammation and accelerate skin ageing. It leads to the production of many biologically active molecules [26-28]. Moreover, this fraction, that contains 
only quercetin, isorhamnetin and kaempferol glycoside derivatives, is not cytotoxic to skin cells unlike the crude extract indicating that toxic molecules were well separated. In conclusion, this fraction could constitute an active and safe natural ingredient usable in cosmetic formulations for its anti-ageing and preservative properties. Results obtained with Kalanchoe Pinnata encourage the use of this semi-preparative HPLC-based activity profiling method to screen other selected plants in our collaborative project.

\section{Acknowledgments}

The authors would like to acknowledge the "Région Centre", "Conseil Général du Loiret" and "Ville d'Orléans" (France) for their financial supports. The authors acknowledged also Christophe Masson (Cosmetic Valley, France) for his advices and Jean-Marc Seigneuret (Adonis, Alban Muller, France) for providing the Kalanchoe pinnata leaves.

\section{Conflicts of Interest}

The authors declare no conflict of interest.

\section{References}

1. Potterat, O.; Hamburger, M. Concepts and technologies for tracking bioactive compounds in natural product extracts: Generation of libraries, and hyphenation of analytical processes with bioassays. Nat. Prod. Rep. 2013, 30, 546-564.

2. Kamboj, A.; Saluja, A.K. Byophyllum pinnatum (Lam.) Kruz;. Phytochemical and pharmacological profile: A review. Pharmacon. Rev. 2009, 3, 364-374.

3. Ojewole, J.A.O. Antinociceptive, anti-inflammatory and antidiabetic effects of Bryophyllum pinnatum (Crassulaceae) leaf aqueous extract. J. Ethnopharmacol. 2005, 99, 13-19.

4. Akinpelu, D.A. Antimicrobial activity of Bryophyllum pinnatum leaves. Fitoterapia 2000, 71, 193-194.

5. Afzal, M.; Gupta, G.; Kazmi, I.; Rahman, M.; Afzal, O.; Alam, J.; Hakeem, K.R.; Pravez, M.; Gupta, R.; Anwar, F. Anti-inflammatory and analgesic potential of a novel steroidal derivative from Bryophyllum pinnatum. Fitoterapia 2012, 83, 853-858.

6. Yadav, N.P.; Dixit, V.K. Hepatoprotective activity of leaves of Kalanchoe pinnata Pers. J. Ethnopharmacol. 2003, 86, 197-202.

7. Almeida, A.P.; da Silva, S.A.; Souza, M.L.; Lima, L.M.; Rossi-Bergmann, B.; Goncalves de Moraes, V.L.; Costa, S.S. Isolation and chemical analysis of a fatty acid fraction of Kalanchoe pinnata with a potent lymphocyte suppressive activity. Planta Med. 2000, 66, 134-137.

8. Pal, S.; Chaudhuri, A.K.N. Studies on the anti-ulcer activity of a Bryophyllum pinnatum leaf extract in experimental animals. J. Ethnopharmacol. 1991, 33, 97-102.

9. Pal, S.; Nag Chaudhuri, A.K. Anti-inflammatory action of Bryophullum pinnatum leaf extract. Fitoterapia 1990, 61, 527-533.

10. Pal, S.; Nag Chaudhuri, A.K. Further studies on the anti-inflammatory profile of the methanolic fraction of the fresh leaf extract of Bryophyllum pinnatum. Fitoterapia 1992, 63, 451-459. 
11. Biswas, S.K.; Chowdhury, A.; Raihan, S.Z.; Muhit, M.A.; Akbar, M.A.; Mowla, R. Phytochemical investigation with assessment of cytotoxicity and antibacterial activities of chloroform extract of the leaves of Kalanchoe pinnata. Am. J. Plant Physiol. 2012, 7, 41-46.

12. Abdellaoui, S.; Destandau, E.; Toribio, A.; Elfakir, C.; Lafosse, M.; Renimel, I.; André, P.; Cancellieri, P.; Landemarre, L. Bioactive molecules in Kalanchoe pinnata leaves: Extraction, purification, and identification. Anal. Bioanal. Chem. 2010, 398, 1329-1338.

13. Yamagishim, T.; Haruna, M.; Yan, X.Z.; Chang, J.J.; Lee, K.H. Antitumor agent; 110. Bryophyllin B, a novel potent cytotoxic bufadienolide from Bryophyllium Pinnatum. J. Nat. Prod. 1989, 52, 1071-1079.

14. Da Silva, S.A.G.; Costa, S.S.; Mendonça, S.C.F.; Silva, E.M.; Moraes, V.L.G.; Rossi-Bergmann, B. Therapeutic effect of oral Kalanchoe pinnata leaf extract in murine leishmaniasis. Acta Trop. 1995, 60, 201-210.

15. Muzitano, M.F.L.; Tinoco, W.; Guette, C.; Kaiser, C.R.; Rossi-Bergmann, B.; Costa, S.S. The antileishmanial activity assessment of unusual flavonoids from Kalanchoe pinnata. Phytochemistry 2006, 67, 2071-2077.

16. Muzitano, M.F.; Falcão, C.A.; Cruz, E.A.; Bergonzi, M.C.; Bilia, A.R.; Vincieri, F.F.; Rossi-Bergmann, B.; Costa, S.S. Oral metabolism and efficacy of Kalanchoe pinnata flavonoids in a murine model of cutaneous leishmaniasis. Planta Med. 2009, 75, 307-311.

17. Nascimento, L.B.S.; Leal-Costa, M.V.; Coutinho, M.A.S.; Moreira, N.D.S.; Lage, C.L.S.; Barbi, N.D.S.; Costa, S.S.; Tavares, E.S. Increased antioxidant activity and changes in phenolic profile of Kalanchoe pinnata (lamarck) Persoon (crassulaceae) specimens grown under supplemental blue light. Photochem. Photobiol. 2013, 89, 391-399.

18. Tatsimo, S.J.N.; Tamokou, J.D.D.; Havyarimana, L.; Csupor, D.; Forgo, P.; Hohmann, J.; Kuiate, J.R.; Tane, P. Antimicrobial and antioxidant activity of kaempferol rhamnoside derivatives from Bryophyllum pinnatum. BMC Res. Notes 2012, 5, 158.

19. Gaind, K.N.; Gupta, R.L. Phenolic compounds from the leaves of Kalanchoe Pinnata. Planta Med. 1973, 23, 149-153.

20. Orth, D.S.; Eck, K.S. Use of triphenyltetrazolium chloride in preservative efficacy testing. Int. J. Cosmet. Sci. 2005, 27, 353.

21. Roehm, N.W.; Rodgers, G.H.; Hatfield, S.M.; Glasebrook, A.L. An improved colorimetric assay for cell proliferation and viability utilizing the tetrazolium salt XTT. J. Immunol. Methods 1991, 142, 257-265.

22. Lebel, C.P.; Ischriropoulos, H.; Bondy, S.C. Evaluation of the probe 2',7'-dichlorofluorescence an indicator of reactive oxygen species formation and oxidative stress. Chem. Res. Toxicol. 1992, 5, 227-231.

23. Jenkins, G. Molecular mechanisms of skin ageing. Mech. Ageing Dev. 2002, 123, 801-810.

24. Chung, H.Y.; Cesari, M.; Anton, S.; Marzetti, E.; Giovannini, S.; Seo, A.Y.; Carter, C.; Yu, B.; Leeuwenburgh, C. Molecular inflammation: Underpinnings of aging and age-related diseases. Ageing Res. Rev. 2009, 8, 18-30.

25. Cuyckens, F.; Claeys, M. Mass spectrometry in the structural analysis of flavonoids. J. Mass Spectrom. 2004, 39, 1-15. 
26. De Vos, S.; Brach, M.; Budnik, A.; Grewe, M.; Herrmann, F.; Krutmann, J. Post-transcriptional regulation of interleukin-6 gene expression in human keratinocytes by ultraviolet B radiation. J. Investig. Dermatol. 1994, 103, 92-96.

27. Aragane, Y.; Yamada, H.; Schwarz, A.; Poppelmann, B.; Luger, T.A.; Tezuka, T. Transforming growth factor-alpha induces interleukin-6 in the human keratinocyte cell line HaCaT mainly by transcriptional activation. J. Investig. Dermatol. 1996, 106, 1192-1197.

28. Suh, D.H.; Kwon, T.E.; Youn, J.I. Changes of comedonal cytokines and sebum secretion after UV irradiation in acne patients. Eur. J. Dermatol. 2002, 12, 139-144.

(C) 2014 by the authors; licensee MDPI, Basel, Switzerland. This article is an open access article distributed under the terms and conditions of the Creative Commons Attribution license (http://creativecommons.org/licenses/by/3.0/). 